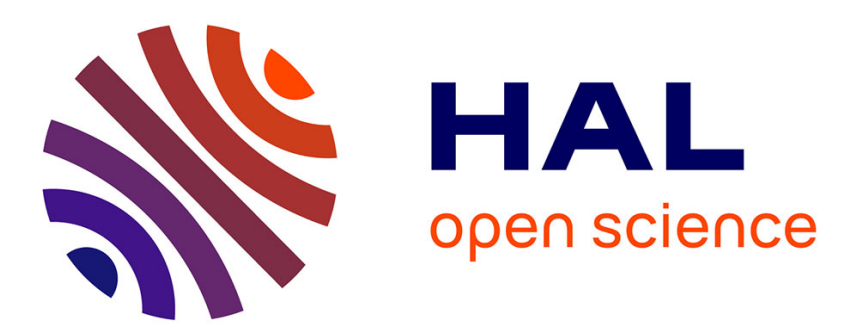

\title{
A model of capillary cohesion for numerical simulations of 3D polydisperse granular media
}

Vincent Richefeu, Moulay Saïd El Youssoufi, Robert Peyroux, Farhang Radjai

\section{To cite this version:}

Vincent Richefeu, Moulay Saïd El Youssoufi, Robert Peyroux, Farhang Radjai. A model of capillary cohesion for numerical simulations of 3D polydisperse granular media. International Journal for Numerical and Analytical Methods in Geomechanics, 2007, 10.1002/nag.674 . hal-00184828

\section{HAL Id: hal-00184828 \\ https://hal.science/hal-00184828}

Submitted on 2 Nov 2007

HAL is a multi-disciplinary open access archive for the deposit and dissemination of scientific research documents, whether they are published or not. The documents may come from teaching and research institutions in France or abroad, or from public or private research centers.
L'archive ouverte pluridisciplinaire HAL, est destinée au dépôt et à la diffusion de documents scientifiques de niveau recherche, publiés ou non, émanant des établissements d'enseignement et de recherche français ou étrangers, des laboratoires publics ou privés. 


\title{
A model of capillary cohesion for numerical simulations of 3D polydisperse granular media
}

\author{
V. Richefeu*, ${ }^{*}$ M. S. El Youssoufi, R. Peyroux and F. Radjaï \\ LMGC, UMR CNRS 5508, Université Montpellier 2, \\ cc 048, Place Eugène Bataillon, F-34095 Montpellier Cedex 5, France
}

\begin{abstract}
SUMMARY
We present a three-dimensional discrete-element approach for numerical investigation of wet granular media. This approach relies on basic laws of contact and Coulomb friction enriched by a capillary force law between particles. We show that the latter can be expressed as a simple explicit function of the gap and volume of the liquid bridge connecting a pair of spherical particles. The length scales involved in this expression are analyzed by comparing with direct integration of the Laplace-Young equation. We illustrate and validate this approach by application to direct shear and simple compression loadings. The shear and compression strengths obtained from simulations reproduce well the experimental measurements under similar material and boundary conditions. Our findings show clearly that the number density of liquid bonds in the bulk is a decisive parameter for the overall cohesion of wet granular materials. A homogeneous distribution of the liquid within the bridge debonding distance, even at low volume contents, leads to highest cohesion. The latter is independent of the liquid content as far as the liquid remains in the pendular state and the number density of liquid bonds remains constant. Copyright (C) 2000 John Wiley \& Sons, Ltd.
\end{abstract}

KEY WORDS: capillary cohesion, discrete element approach, shear strength, compression strength

\section{INTRODUCTION}

Granular materials are composed of well-defined particles with specific kinematics locally dictated by steric constraints and unilateral interactions that are responsible for the rich behavior of these materials at the macroscopic scale. These materials can thus be modeled at the particle scale, and various particle properties (shape, size... ) and contact interactions (friction, adhesion... ) can be quite naturally introduced in discrete element numerical simulations of the material. In the same way, external and environmental factors such as moisture and temperature may be included in the description through dependence of local parameters with respect to such factors. In spite of drawbacks, mainly related to unavoidable simplification of the local description and the number of particles limited by computational efficiency, this approach with the corresponding numerical developments provides new scopes for a

*Correspondence to: V. Richefeu, LMGC, Université Montpellier 2, cc 048, Place Eugène Bataillon,

F-34095 Montpellier Cedex 5, France.

${ }^{\dagger}$ E-mail: richefeu@1mgc.univ-montp2.fr 
better understanding of the behavior of complex materials from their rich microstructural properties. The discrete element method (DEM) for the simulation of dry granular media, first popularized by the pioneering work of Cundall [1], has evolved into a mature technique during the two last decades $[2,3,4,5]$. The focus is now mostly directed towards the inclusion of new ingredients such as cohesion due to cementation or capillary bonding as in unsaturated soils $[6,7,8,9]$.

This paper is concerned with the mechanical behavior of wet granular materials investigated both by a discrete element approach and experiments. Wet granular materials are of primary interest to various fields of science of engineering such as the mechanics of granular soils and wet processing of powders $[10,11]$. The capillary cohesion is negligibly small for coarse soils or at high confining stresses. On the other hand, the moisture and the resulting cohesion are important for fine surface soils. For example, when plowing a wet granular soil, large cohesive aggregates are formed. The largest capillary cohesion force for millimeter-size sand grains is about $4 \times 10^{-4} \mathrm{~N}$ independently of the volume of the capillary bond. This force is nearly four times the grain weight, allowing thus for the formation of cohesive aggregates. Transformations involving primary particle agglomeration into coherent granules are of special interest in many applications in a wide range of industries such as pharmaceuticals, agronomic products and detergents $[10,11]$.

Recently, several simulations of wet granular media have been reported [12, 13, 14]. Mikami et al. [15] used this type of simulation together with a regression expression for the liquid bridge force as a function of liquid bridge volume and separation distance between particles. They mainly studied bubbling behavior and agglomerate formation in a fluidized bed and they found realistic results. Dense agglomerates were simulated by Gröger et al. [16] using a cohesive discrete element method. They found a good agreement with experimental data for the yield stress at all confining pressures down to the value of the tensile stress. Shear strength behavior of unsaturated granulates was also studied numerically by Jiang et al. [17] as a function of suction (pressure difference between liquid and gas).

From the experimental point of view, the point is that classical testing machines employed in soil mechanics are designed to work at high levels of confinement and they involve massive elements that induce high inertia. For these reasons, they are not adapted for wet granular materials. Direct measurements of tensile strength by means of appropriate experimental setups have been reported recently $[18,19,20]$. In granular media, it is generally much more difficult to access local information such as contact forces or liquid bonds. Few investigations have recently been reported to visualize liquid bonds by means of the index matching technique [21, 22].

Hence, both the numerical implementation of capillary interactions and the use of appropriate testing techniques are key aspects of the present work. In this paper, we present a new expression for the capillary force as an explicit function of the interparticle gap and local volume of the liquid. We show that this expression provides excellent fit for the capillary force between two particles of unequal sizes. This expression is used to perform DEM simulations of direct shear and simple compression tests, which are compared with experimental data obtained by means of an appropriate testing setup. In the following, we first present in Section 2 the numerical approach with focus on capillary cohesion. In Section 3, we apply the method to direct shearing of wet granular samples. The main characteristics of our experimental setup designed for low confining stress are described in this section. We compare the numerical and experimental data, and we analyze the effect of water content and the numerical density of capillary bonds. A similar approach is presented in Section 4 in the case of simple compression tests which confirm the good agreement between numerical and experimental data as to stress-strain behavior and the effect of liquid distribution. We conclude with a summary of the main results and perspectives of this work. 


\section{NUMERICAL METHOD}

We used the discrete element method (DEM) in the spirit of molecular dynamics with a velocity Verlet integration scheme [1,23]. In DEM simulations, the equations of motion of the particles are integrated incrementally by taking into account the interactions between the particles according to an explicit molecular-dynamics-like scheme. The realism of such simulations is thus dependent on the underlying model of the interactions. For numerical stability, the time step was set to $10 \%$ below the elastic response time $\Delta t_{c}=\pi \sqrt{m / K_{n}}$, where $m$ is the smallest particle mass and $K_{n}$ is the largest normal stiffness in the system $[1,23]$. In quasistatic loadings, where the inertia plays no major role, the particle masses can be increased artificially, allowing thus for larger time steps. On the other hand, the damping rate $v_{n}$ should be below the critical value $v_{c r i t}=2 \sqrt{m K_{n}}$. In this section, we first present the interaction laws that we used for elastic contact, Coulomb friction and capillary cohesion. We discuss in more detail a new analytical form that we propose for the capillary force as a function of the gap and local water volume. Then, we present the protocols for deriving the distribution of liquid in the bulk.

\subsection{Normal repulsion force}

The force laws involve normal repulsion, capillary cohesion, Coulomb friction, and normal damping. The normal force $f_{n}$ is modeled as a sum of three contributions:

$$
f_{n}=f_{n}^{e}+f_{n}^{d}+f_{n}^{c} .
$$

where $f_{n}^{e}, f_{n}^{d}$ and $f_{n}^{c}$ are the repulsive contact force, damping force and capillary force, respectively.

The repulsive force between two smooth elastic spheres is given by the Hertz approximation which expresses the repulsion force as a function of the distance between two spheres [24]. From a computational viewpoint, it is more common to use a linear approximation where $f_{n}^{e}$ depends linearly on the normal distance $\delta_{n}$ between the particles (Fig 1(a)):

$$
f_{n}^{e}=\left\{\begin{array}{ll}
-k_{n} \delta_{n} & \text { for } \quad \delta_{n}<0 \\
0 & \text { for } \quad \delta_{n} \geq 0
\end{array},\right.
$$

where $k_{n}$ is the normal stiffness.

The damping term $f_{n}^{d}$ accounts for inelastic shock between particles. The simplest model of damping is a viscous force depending linearly on the normal velocity $\dot{\delta}_{n}$ :

$$
f_{n}^{d}=\left\{\begin{array}{ll}
2 \alpha_{n} \sqrt{m k_{n}} \dot{\delta}_{n} & \text { for } \quad \delta_{n}<0 \\
0 & \text { for } \quad \delta_{n} \geq 0
\end{array},\right.
$$

where $m=m_{i} m_{j} /\left(m_{i}+m_{j}\right)$ is the reduced mass of the particles $i$ and $j, \alpha_{n}$ is a damping rate varying in the range $[0,1[$. The rate of normal dissipation or the restitution coefficient between particles can be expressed as a function of $\alpha_{n}$ [25].

The repulsion force $f_{n}^{e}$ together with the viscous damping force $f_{n}^{d}$ define a spring-dashpot model commonly used for the simulation of dry granular media. Subtle changes to this framework allow for more realistic description of shock laws and nonlinear elastic regimes [25]. Since we are interested here in capillary interactions, we stay with this basic framework and focus on the capillary force $f_{n}^{c}$.

\subsection{Capillary force}

The capillary force $f_{n}^{c}$ is a function of the liquid bond parameters, namely the gap $\delta_{n}$, the liquid bond volume $V_{b}$, the liquid surface tension $\gamma_{s}$, and the particle-liquid-gas contact angle $\theta$. The capillary 

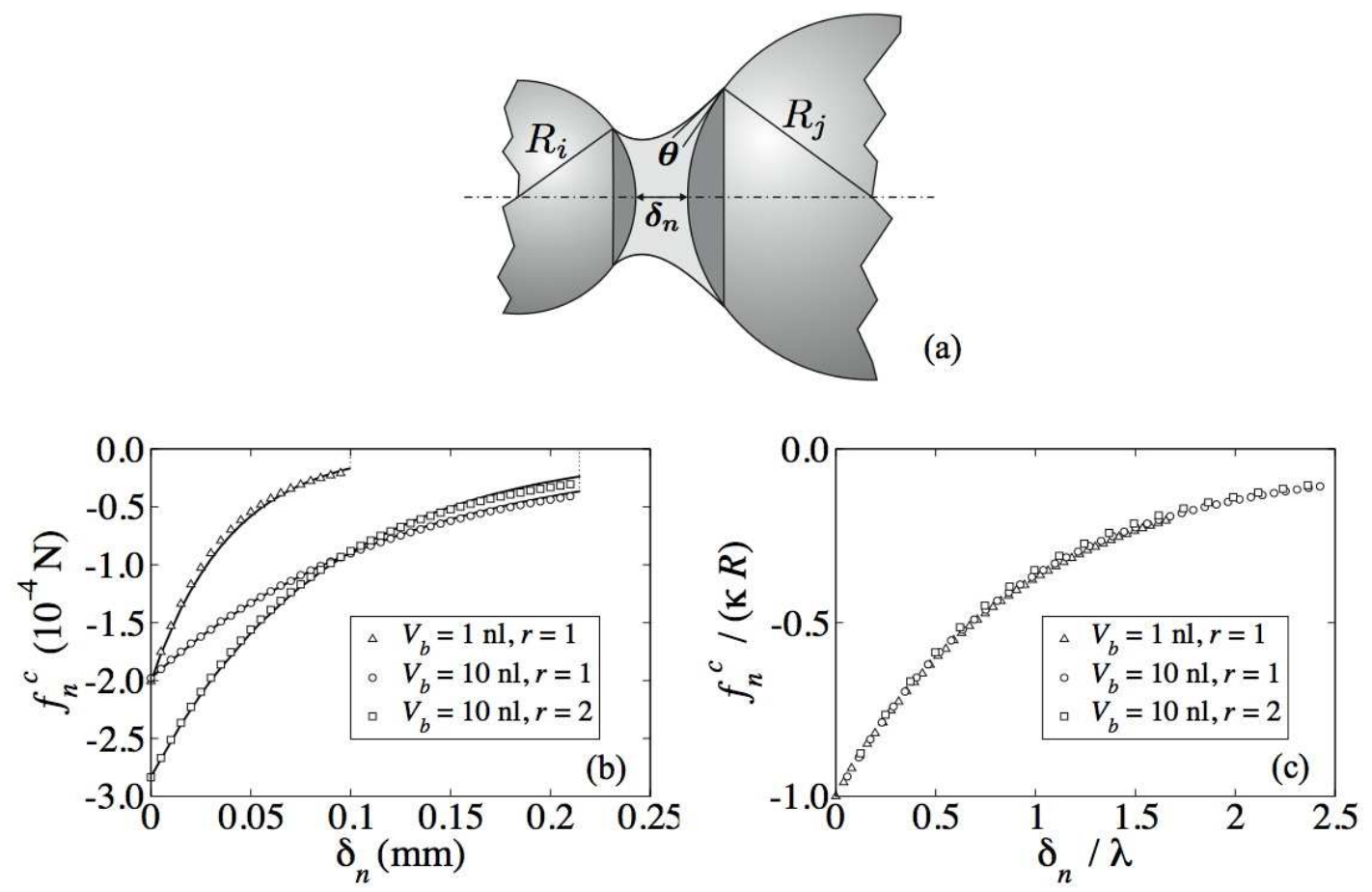

Figure 1. (a) Geometry of a capillary bridge; (b) Capillary force $f_{n}^{c}$ as a function of the gap $\delta_{n}$ between two particles for different values of the liquid volume $V_{b}$ and size ratio $r$ according to the model proposed in this paper (solid lines), and from direct integration of the Laplace-Young equation (open circles); (c) Scaled plot of the capillary force as a function of gap from the direct data shown in (b).

force can be obtained by integrating the Laplace-Young equation [15, 26, 27, 28]. However, for efficient DEM simulations, we need an explicit expression of $f_{n}^{c}$ as a function of the liquid bond parameters. On the other hand, most authors have considered the capillary force for liquid bond between two spheres of the same diameter. When the diameters are different the Derjaguin approximation is used. Recently, by means of experiments and fitting considerations, Soulie et al. [28] proposed an expression for the capillary force between two smooth spheres. Here, we propose a new expression for the capillary force which can be considered as a simplified and analytical writing of that expression. We show that this form is well fitted by the data from direct integration of the Laplace-Young equation both for monodisperse and polydisperse particles.

The geometry of a capillary bridge between two spherical particles of unequal diameters is illustrated in Figure 1(a). At leading order, the capillary force $f_{0}$ at contact, i.e. for $\delta_{n} \leq 0$, is

$$
f_{0}=-\kappa R,
$$

where $R$ is a length depending on the particle radii $R_{i}$ and $R_{j}$ and $\kappa$ is given by [29,30,31]

$$
\kappa=2 \pi \gamma_{s} \cos \theta .
$$

Note that a negative value of $\delta_{n}$ corresponds to an overlap between the particles. The assumption is that the overlap is small compared to the particle diameters. The data obtained from direct integration of the 
Laplace-Young equation show that the geometric mean $R=\sqrt{R_{i} R_{j}}$ is more suited than the harmonic mean $2 R_{i} R_{j} /\left(R_{i}+R_{j}\right)$ proposed by Derjaguin for polydisperse particles in the limit of small gaps (see below) [32]. We also note that $f_{0}$ in Equation (4) is independent of the bond liquid volume $V_{b}$.

The adhesion force $f_{0}$ at contact is the highest level of the capillary force. The latter declines as the gap $\delta_{n}$ increases. The capillary bridge is stable as long as $\delta_{n}<\delta_{n}^{\max }$, where $\delta_{n}^{\max }$ is the debonding distance given by [13]

$$
\delta_{n}^{\max }=\left(1+\frac{\theta}{2}\right) V_{b}^{1 / 3} .
$$

We note that the debonding distance depends only on the liquid volume $V_{b}$ whereas the adhesion force $f_{0}$ at contact is a function only of particle diameters through the geometric mean $R$.

Between these two limits, the capillary force falls off exponentially with $\delta_{n}$ :

$$
f_{n}^{c}=f_{0} e^{-\delta_{n} / \lambda}
$$

where $\lambda$ is a length scale which should be a function of $V_{b}$ and the particle radii. The role of particle size is two-fold. On one hand, the liquid volume should be compared to a mean particle radius $R^{\prime}$, a function of $R_{i}$ and $R_{j}$, but which can be different from $R$ introduced in Equation (4) for the adhesion force. On the other hand, the asymmetry due to unequal particle sizes can be taken into account through a function of the ratio between particle radii. We set

$$
r=\max \left\{R_{i} / R_{j} ; R_{j} / R_{i}\right\}
$$

Dimensionally, a plausible expression of $\lambda$ is

$$
\lambda=c h(r)\left(\frac{V_{b}}{R^{\prime}}\right)^{1 / 2}
$$

where $c$ is a constant and $h$ is a function only of $r$. When introduced in Equations (9) and (7), this form yields a nice fit for the capillary force obtained from direct integration of the Laplace-Young equation by setting $R^{\prime}=2 R_{i} R_{j} /\left(R_{i}+R_{j}\right), h(r)=r^{-1 / 2}$ and $c \simeq 0.9$.

Figure 1(b) shows the plots of Equation 7 for three different values of the liquid volume $V_{b}$ and size ratio $r$ together with the corresponding data from direct integration. We see that the fit is excellent at $\delta_{n}=0$ (at contact) and for nearly all values of $\delta_{n}$ up to the debonding distance. Figure 1(c) shows the same plots of the direct data as in Figure 1(b) but where the forces are normalized by $\kappa R$ and the lengths by $\lambda$. The data collapse on the same plot, indicating again that the force $\kappa R$ and the expression of $\lambda$ in Equation (9) characterize correctly the behavior of the capillary bridge.

In summary, the capillary cohesion can be expressed in the following form:

$$
f_{n}^{c}= \begin{cases}-\kappa R & \text { for } \delta_{n}<0 \\ -\kappa R e^{-\delta_{n} / \lambda} & \text { for } 0 \leq \delta_{n} \leq \delta_{n}^{\max } \\ 0 & \text { for } \delta_{n}>\delta_{n}^{\max }\end{cases}
$$

with

$$
\lambda=\frac{c}{\sqrt{2}} V_{b}^{\frac{1}{2}}\left\{\max \left(R_{i} / R_{j} ; R_{j} / R_{i}\right)\right\}^{-\frac{1}{2}}\left\{\frac{1}{R_{i}}+\frac{1}{R_{j}}\right\}^{\frac{1}{2}} .
$$

In the simulations, a capillary bridge is removed as soon as the debonding distance is reached, and the liquid is redistributed among the contacts belonging to the same particle in proportion to grain diameters [33]. We also assume that the particles are perfectly wettable, i.e. $\theta=0$. This is a good approximation for water and glass beads. 


\subsection{Friction force}

For the friction force $\mathbf{f}_{t}$, we use the well-known viscous-regularized Coulomb law $[25,34,35]$,

$$
\mathbf{f}_{t}=-\min \left\{\gamma_{t}\left\|\dot{\boldsymbol{\delta}}_{t}\right\|, \mu\left(f_{n}-f_{n}^{c}\right)\right\} \frac{\dot{\boldsymbol{\delta}}_{t}}{\left\|\dot{\boldsymbol{\delta}}_{t}\right\|},
$$

where $\gamma_{t}$ is a tangential viscosity parameter, $\mu$ is the coefficient of friction, and $\dot{\boldsymbol{\delta}}_{t}$ is the sliding velocity. In relaxation to equilibrium, $\dot{\delta}_{t}$ declines but never vanishes due to residual kinetic energy. The equilibrium state is practically reached as soon as we have $\gamma_{t}\left\|\dot{\boldsymbol{\delta}}_{t}\right\|<\mu\left(f_{n}-f_{n}^{c}\right)$ at all contacts, i.e. when the friction force is inside the Coulomb cone everywhere in the system.

\subsection{Distribution of liquid}

The capillary force $f_{n}^{c}$, according to Equation (10), and the debonding distance $\delta_{n}^{\max }$ in Equation (6) depend on the bond liquid volume $V_{b}$. It is thus important to use a convenient distribution rule for the allocation of the total volume $V_{\ell}$ of the liquid to contacts or adjacent particles within the debonding distance. To do so, the following conditions must be satisfied:

(i) The liquid is fully distributed in the form of capillary bonds (no liquid at the interstitial sites or pores), so that

$$
V_{\ell}=\sum V_{b}
$$

(ii) The bond volume is dependent on the mean particle size. This is because the liquid retention capacity increases with particle size.

(iii) The particle pairs with a gap beyond the debonding distance are not eligible to receive liquid.

For a homogeneous distribution, the liquid is attributed to all eligible pairs. We assume that the bond volumes are proportional to the volumes of the pairs:

$$
V_{b}=\alpha R^{3},
$$

where $\alpha$ is fixed by the condition (13). The use of geometric mean $R \equiv \sqrt{R_{i} R_{j}}$ is a simple matter of choice. Any other mean can be used for the same purpose. When debonding occurs, the bond liquid volume is redistributed among neighboring bonds. This local redistribution can gradually lead to a globally inhomogeneous distribution. In order to ensure homogeneous distribution all along a simulation run, the liquid distribution should be updated regularly according to Equation (14). As a result, new eligible pairs appear and new liquid bonds are formed. Below, we will refer to this redistribution procedure as Protocol 1.

The distribution can be made more realistic by taking into account the "loss" of liquid in the form of droplets at the surface of the particles or partial draining due to gravity. The liquid may also cluster in the interstitial pores [21, 22, 36]. However, most simulation results presented below are not sensitive to such refinements. This is because the deformations are not large enough for the liquid distribution to evolve significantly from the initial homogeneous distribution. 


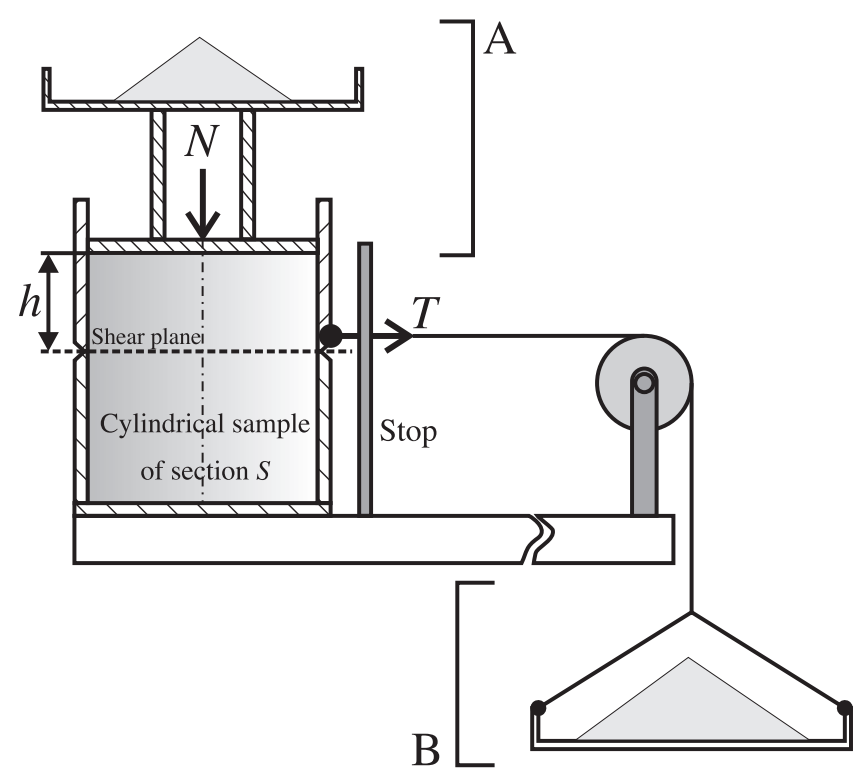

Figure 2. Testing cell and shearing setup.

\section{DIRECT SHEAR}

\subsection{Experiments}

We designed an experimental setup which, in contrast to the standard Casagrande testing machine, allowed us to measure the shear strength at very low confining pressures $(<1 \mathrm{kPa})$. Similar setups have been used by several authors in the past [37, 38, 39, 40]. We present here the setup, the materials and the wetting protocol and our main experimental results.

3.1.1. Experimental setup A sketch of the shearing setup is shown in Figure 2. Wetted grains are poured in a plexiglas cylindrical cell and confined by means of a circular lid of area $S$ placed on top of the material. The lid is equipped with a reservoir allowing to impose an overload by adding desired amount of sand. The total vertical force $N$ acting on the sample is the sum of the weights of lid and sand (A). The cell is composed of two disjoint parts kept together during sample preparation. The upper part can move horizontally with respect to the lower part by pulling on a rope attached to it and which supports a cupel through a pulley (B). The pulling force $T$ can be increased by adding sand into the cupel. The friction force between the two parts of the cell is reduced by water lubricating the rims. In order to reduce the friction force exerted by the material along the walls, the thickness $h$ of the upper part of the sample is taken to be below the diameter of the cell $(46 \mathrm{~mm})$. The heights of the upper and lower parts are about $10 \mathrm{~mm}$ and $15 \mathrm{~mm}$ respectively.

The sample is sheared along the common section of the two parts of the cell. This shear plane is subjected to a tangential stress $\tau=T / S$ and a normal stress $\sigma=N / S+\rho g h$, where $\rho$ is the bulk density and $g$ the gravity. We gradually increase the shear stress $\tau$ for $\sigma$ kept constant. Unstable failure occurs when $\tau$ reaches the shear strength $\tau_{m}$. At this point, an infinitesimal stress increment causes a 
finite deformation of the sample manifesting itself as a sudden slide of the upper part of the sample. The upper part is stopped by collision with two bars located $5 \mathrm{~mm}$ away from the cell. We did not measure the displacements. We recorded $\tau_{m}$ for different values of $\sigma$ in the range varying from $200 \mathrm{~Pa}$ to $800 \mathrm{~Pa}$, and for different values of water content.

3.1.2. Materials and wetting protocol Four types of materials were tested: (1) a sand "S" composed of angular grains with diameters ranging from $0.1 \mathrm{~mm}$ to $0.4 \mathrm{~mm}$, (2) "tightly-graded" polydisperse glass beads "GB45" with diameters from $0.4 \mathrm{~mm}$ to $0.5 \mathrm{~mm}$, (3) "well-graded" polydisperse glass beads "GB48" with diameters from $0.4 \mathrm{~mm}$ to $0.8 \mathrm{~mm}$, and (4) monodisperse glass beads "GB1" of diameter $1 \mathrm{~mm}$.

The grains were wetted by adding distilled water to dry material placed in a vessel and shaking energetically until all visible water clusters disappear. The vessel used for mixing is transparent allowing us to check visually the state of the material. After mixing, the wetted material is poured into the testing cell. The water content is evaluated by comparing the masses of a sample of the material before and after testing by means of a heat chamber used for drying the sample at $105^{\circ} \mathrm{C}$. The water content is given by $w=m_{w} / m_{s}$, where $m_{w}$ and $m_{s}$ are the masses of water and grains, respectively. The wet materials were tested for water contents below $5 \%$ corresponding to the pendular state for our materials. The experiments were performed at ambient conditions. Each experiment lasted a few minutes. The loss of liquid was always below two percents. This loss is not only due to evaporation but also to partial wetting of the internal walls of the cell. But it is low enough to assume a constant liquid volume (as in simulations, see below).

3.1.3. Results Several tests were carried out with the four materials at our disposal (S, GB45, GB48 and GB1) for different values of water content $w$ and normal stress $\sigma$. Figure 3 shows the yield loci $\tau-\sigma$. Within experimental precision, the data are well fitted by a straight line for each material, in agreement with the Mohr-Coulomb model

$$
\tau=(\tan \varphi) \sigma+c,
$$

where $\tan \varphi$ is the internal coefficient of friction and $c$ is the Coulomb cohesion. We also observe that the angle of internal friction $\varphi$ is independent of $w$ as the Coulomb lines are nearly parallel.

Using a similar experimental setup, Schellart found that the yield loci are curved down as the normal stress tends to zero [38]. In dry granular media, the shear stress vanishes naturally at zero normal stress. However, in the wet case according to Figure 3, there is a finite cohesion $c$ corresponding to the intersection point of the Coulomb line with the axis $\sigma=0$. Figure 4 displays the evolution of $c$ as a function of $w$ for the four materials. The evolution of $c$ is strongly nonlinear and saturates at a level $c=c_{m}$ for a water content $w=w_{m}$, both depending on the material. The experimental estimations of $c_{m}$ and $w_{m}$, as well as the internal angle of friction for our materials are given in Table I.

\begin{tabular}{llllr}
\hline & $\langle D\rangle$ & $\varphi$ & $c_{m}$ & $w_{m}$ \\
\cline { 2 - 5 } S & $0.16 \mathrm{~mm}$ & $33^{\circ}$ & $600 \mathrm{~Pa}$ & $3 \%$ \\
GB45 & $0.45 \mathrm{~mm}$ & $30^{\circ}$ & $350 \mathrm{~Pa}$ & $2.5 \%$ \\
GB48 & $0.6 \mathrm{~mm}$ & $30^{\circ}$ & $300 \mathrm{~Pa}$ & $1 \%$ \\
GB1 & $1 \mathrm{~mm}$ & $25^{\circ}$ & $150 \mathrm{~Pa}$ & $<1 \%$ \\
\hline
\end{tabular}

Table I. Characteristic data from experimental measurements. 


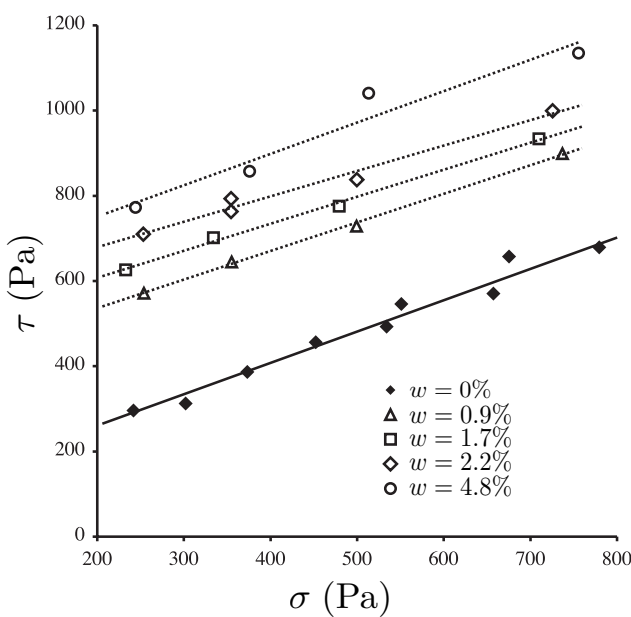

(a) $\mathrm{S}$

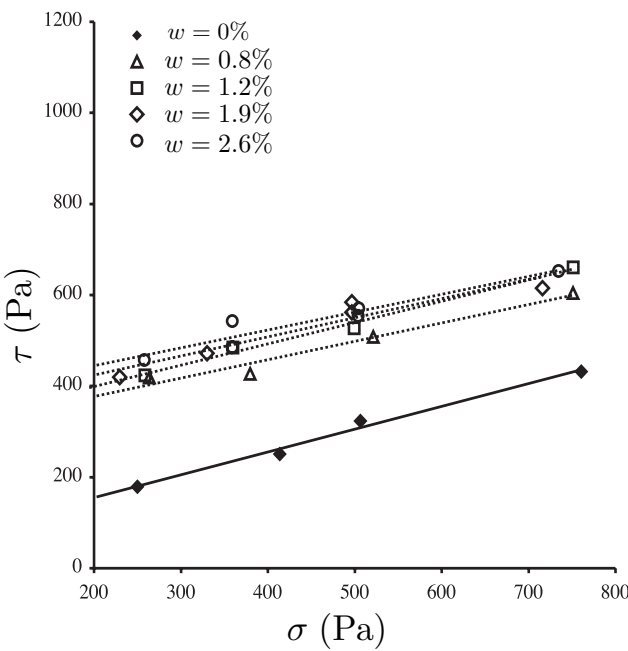

(c) GB48

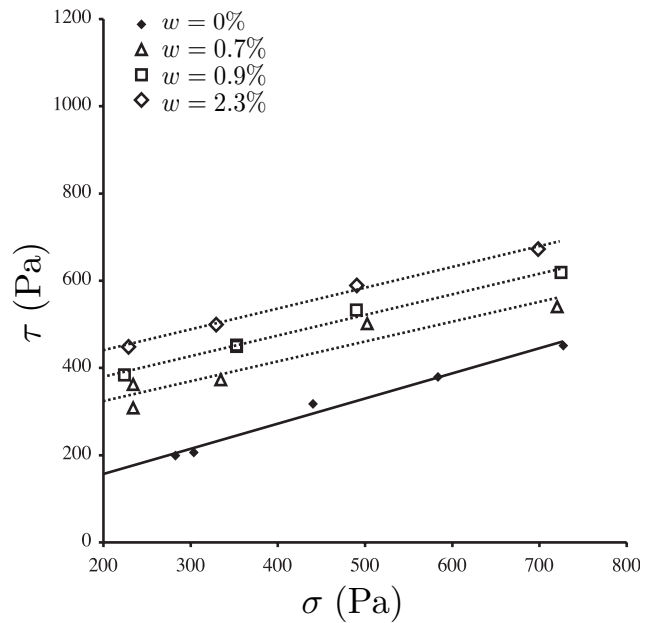

(b) GB45

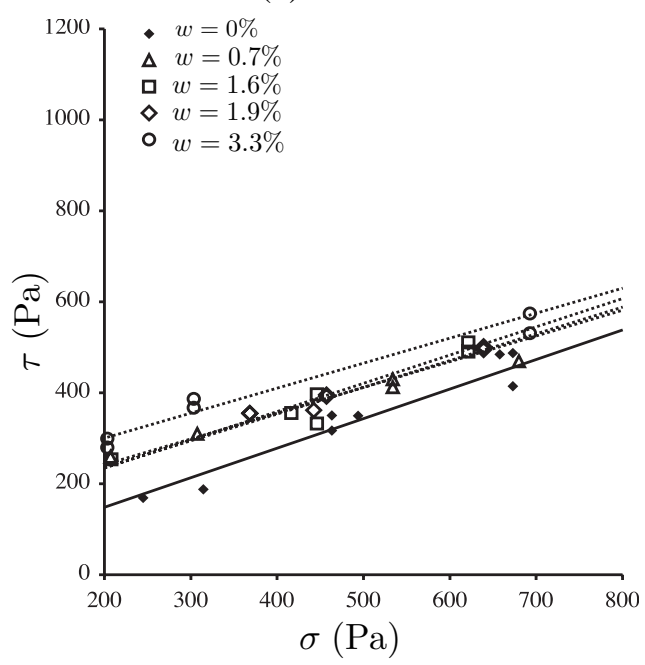

(d) GB1

Figure 3. Yield loci, fitted by straight lines, for our experimental granular materials.

The experimental data at different levels of water content show larger fluctuations for glass beads (GB) than for sand. These fluctuations stem certainly from the lower level of cohesion for glass beads and the also from their tighter particle size distribution. The differences in the values of $c_{m}$ can be attributed to differences in the mean particles sizes in different materials. In the case of sand, the nonspherical form of the particles might also affect the value of $c_{m}$. The value of $w_{m}$ is less clearly defined and is likely to depend on the surface state of the particles [36]. The sand grains have a rough surface requiring more water to form a meniscus than the more smooth glass beads. On the other hand, partial clustering of water may occur and this might require a larger amount of water for the formation of liquid bridges [21, 22]. 


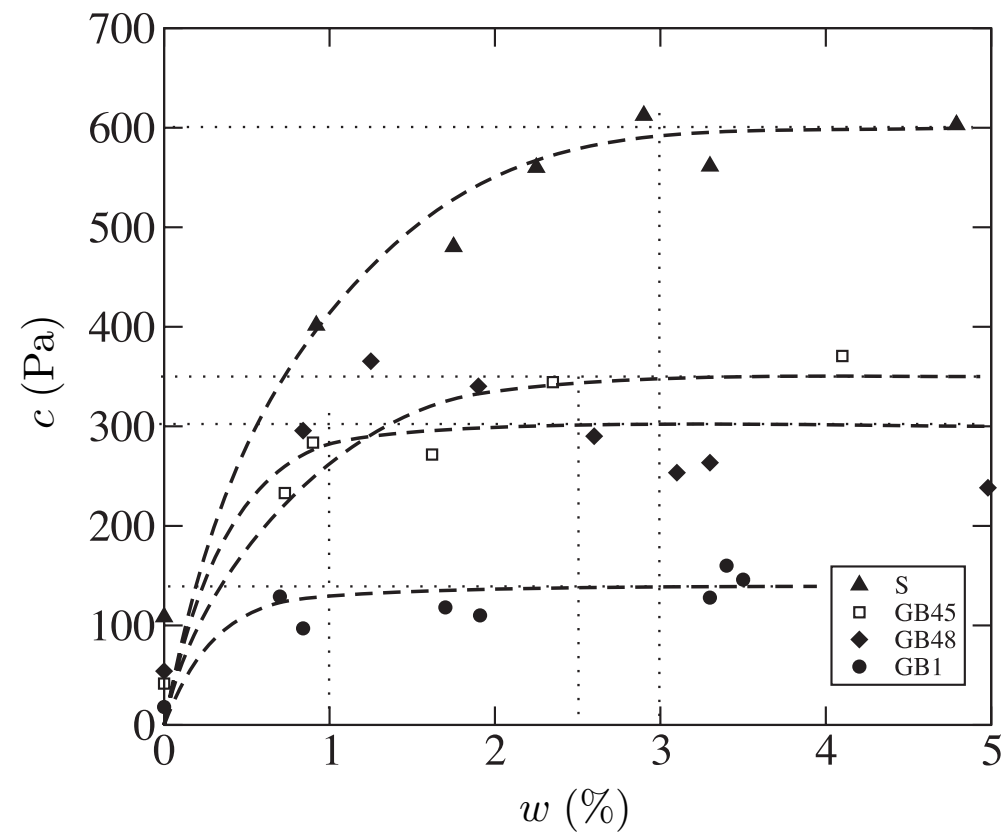

Figure 4. Macroscopic cohesion $c$ as a function of water content $w$ for four tested granular materials. The trends are represented by dashed lines as a guide to the eyes.

\subsection{Numerical simulations}

3.2.1. Sample preparation The numerical samples are composed of 7307 spherical particles of diameters $2 \mathrm{~mm}, 1.5 \mathrm{~mm}$ and $1 \mathrm{~mm}$ in proportions of $50 \%, 30 \%$ and $20 \%$, respectively. The dimensions and the total volume of the numerical sample are similar to those of the experimental samples. This numerical sample can be compared to the sample GB1 of glass beads (Table I) although the size distribution of the particles is slightly different. In fact, using exactly the same particle sizes as in experiments with the same total volume would require many more particles in simulations and thus much more computation time. The particles are placed randomly in a cylindrical cell. The initial configuration is prepared under gravity without introducing capillary bonds. Then, we attribute a capillary bond to eligible pairs of particles (within the de-bonding distance). Finally, the sample is consolidated under the action of a vertical confining pressure with a zero coefficient of friction. The consolidation is stopped and the coefficient of friction set to 0.4 as soon as the solid fraction $\phi=0.6$ is reached. The subsequent compaction is negligibly small.

As in experiments, the cylindrical cell is composed of two disjoint parts. The lower part is fixed whereas the upper part moves horizontally, giving rise to a shear plane along the common section of the two parts. We apply a constant vertical load $\sigma$, the same as in experiments, on top of the sample. However, in contrast to experiments, shearing is controlled by imposing a constant horizontal velocity on the upper part. The numerical sample has exactly the same dimensions as in experiments.

During shearing, the number of liquid bonds evolves and the available liquid must be redistributed in the system. We used two different methods for redistribution: (1) we simply apply the above procedure every time the contact list is updated (Protocol 1); (2) the volume of a broken liquid bond is split 
between the corresponding particles (proportionally to their diameters) and conserved for the formation of new liquid bonds when a contact occurs with the same particles (Protocol 2). In this method, the volume of free liquid left after de-bonding is kept with the two particles (and not distributed to the other bonds of the same particles) and used only if a new contact is formed. This implies that, if the initial liquid distribution is homogeneous, then it will remain so during deformation as in the first method. In other words, the liquid will not migrate considerably and one should expect quite similar results from both methods. Indeed, in different tests, we found that both methods lead to nearly identical results (see Figure 12 in Section 4.2.2).

3.2.2. Numerical results and comparison with experiments We performed a series of direct shear simulations with different values of the water content from $0 \%$ to $2 \%$.

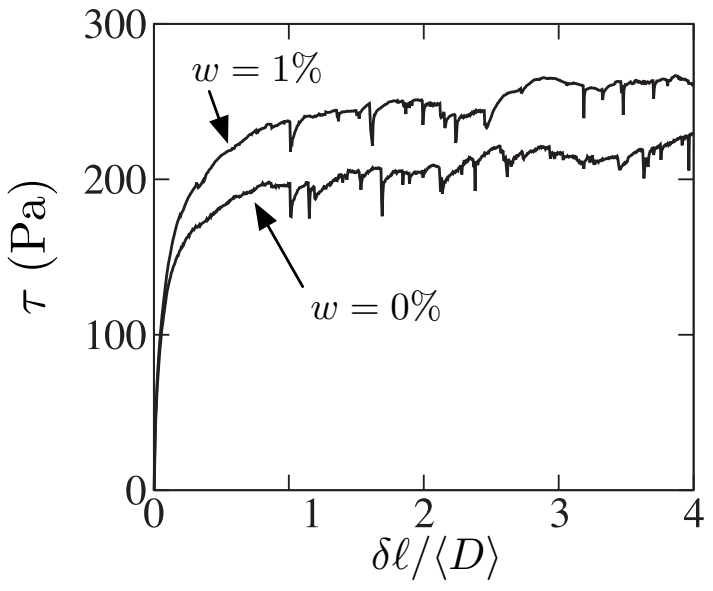

Figure 5. Stress-strain plot for $w=0 \%$ and $w=1 \% ; \sigma=300 \mathrm{~Pa}$. The displacements are normalized by the average particle diameter $\langle D\rangle$.

Figure 5 shows the shear strain $\tau$ for a dry and a wet sample with $w=1 \%$ as a function of horizontal displacement $\delta \ell$. The initial configuration is the same in both simulations. The residual state is reached without passing by a stress peak and for a displacement of the order of one particle diameter for all tested values of the water content. The steady state deformation involves numerous instabilities that occur throughout the system and appear in the form of rapid stress drops on the stress-strain plots. We see that in transition from dry to wet materials, the frequency of such instabilities declines.

The evolution of the Coulomb cohesion can be analyzed as a function of water content $w$ as in experiments. Figure 6(a) shows fitted yield loci from 15 simulations with three different values of the confining pressure $\sigma$ and five different values of $w$. The Coulomb cohesion $c$ is drawn as a function of $w$ in Figure 6(b). The latter is very similar to the corresponding experimental plot (Figure 4(c)) for monodisperse glass beads. We observe a saturation of $c$ at still lower levels of water content $\left(w_{m} \simeq 0.1 \%\right)$.

The limit value $c_{m}$ of the Coulomb cohesion as a function of water content, as observed here both in the simulations and experiments, is not intuitive. Although the liquid bond volume appears in Equations (6) and (11), it is important to remark that failure is initiated at contacts where the maximum capillary force $f_{0}$ is reached and this force is independent of the local liquid volume. For this reason, the Coulomb cohesion is mainly controlled by the density of liquid bonds or equivalently by the bond 


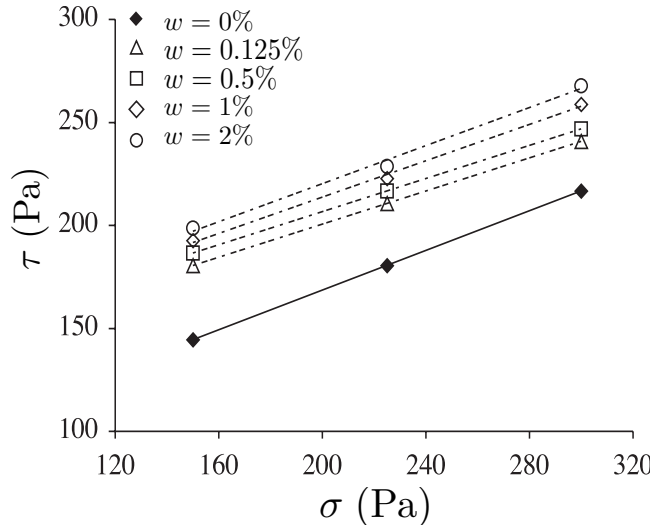

(a)

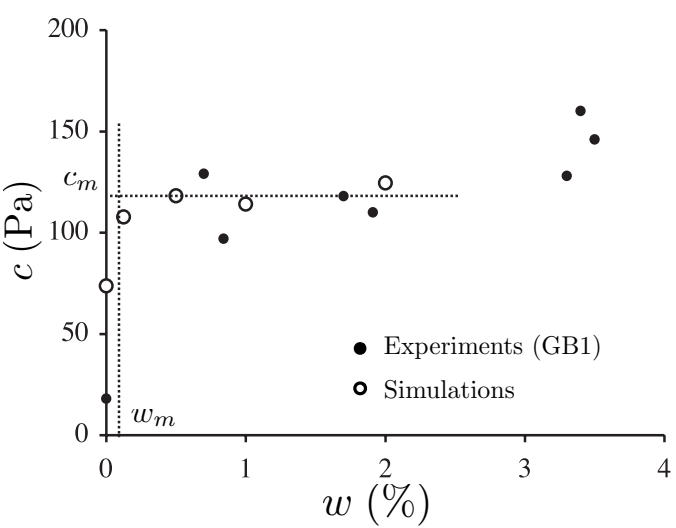

(b)

Figure 6. Simulation results. (a) Estimated yield loci. (b) Coulomb cohesion as a function of water content (experiments were carried out with $1 \mathrm{~mm}$ glass beads).

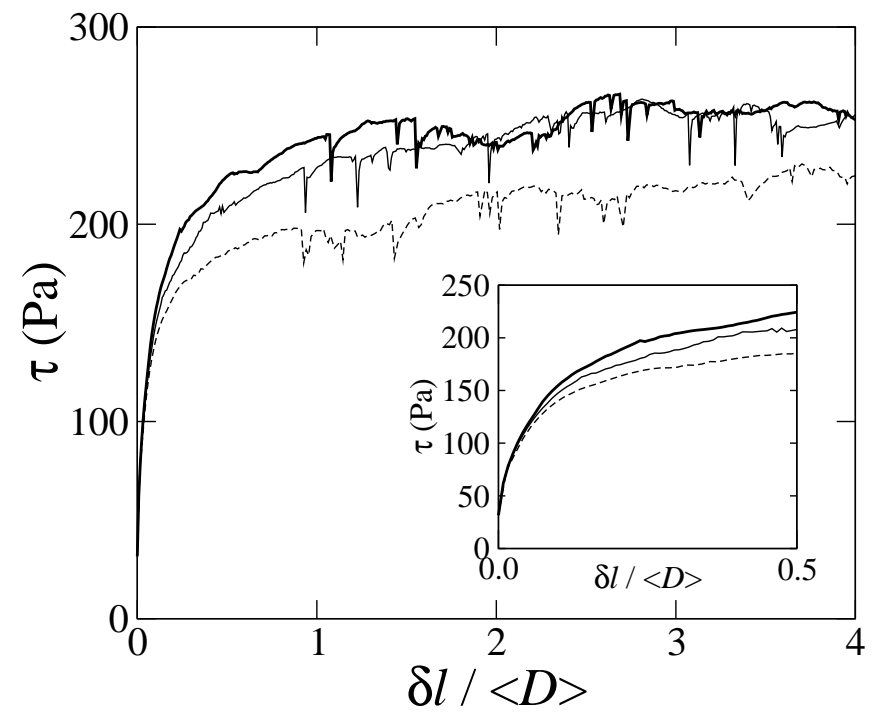

Figure 7. The shear stress $\tau$ as a function of shearing distance $\delta \ell$ normalized by the average particle diameter $\langle D\rangle$ for a dry (dashed line) and two wet samples (solid lines) with a high bond coordination number (thick line) and with two times less bonds (thin line). The inset shows a zoom for small deformations.

coordination number $z$. In order to illustrate the effect of $z$, in Figure 7 we show the stress-strain plots for two samples differing in the number of liquid bonds for the same water content. The initial particle configuration is the same in both samples but there are two times less bonds in one sample (obtained by removing half of the bonds in the first sample). We see that in the initial stages of deformation, the cohesion is close to half that of the sample involving a double number of water bonds, and it increases as the wet coordination number grows. The Coulomb cohesion saturates when the bond 
coordination number saturates as the total liquid volume is increased. This means that the main effect of the liquid volume (factor $V_{b}^{1 / 3}$ in Equation 6) is to increase the debonding distance $\delta_{\text {max }}$ and thus the bond coordination number as liquid volume is increased in the sample. The bond coordination number saturates when each particle has already a bond with all of its first neighbors.

We observe that the maximum cohesion $c_{m}=120 \mathrm{~Pa}$ in the simulations is below that $\left(c_{m}=150\right.$ $\mathrm{Pa}$ ) for $1 \mathrm{~mm}$ glass beads. The lower value of $c_{m}$ in simulations can be attributed to the larger average diameter of the particles compared to experimental samples. Indeed, it can be shown that the Coulomb cohesion varies in inverse proportion to the average particle size [33]. This effect of the particle size can also be observed for other experimental samples in Figure 4 where $c_{m}$ and $w_{m}$ are lower for larger values of $\langle D\rangle$.

\section{SIMPLE COMPRESSION}

In this section we consider the influence of water content on the strength of our granular samples under simple compression loading. The strains are known to be more homogeneous in compression than in direct shear. On the other hand, the strengths are weaker and thus the capillary effects are straightforward to measure. The experimental data are borrowed from Soulié [41] who compared also his results with numerical simulations and found qualitative agreement between them. He explained the quantitative differences of numerical data with experimental data by invoking the distribution of water. We will revisit below the same data in the light of our new numerical developments.

\subsection{Review of experimental results}

The experimental tests were carried out with samples of glass beads of diameters ranging from $0.8 \mathrm{~mm}$ to $1.3 \mathrm{~mm}$. The samples were of cylindrical shape with a diameter of $25 \mathrm{~mm}$ and a height of $17 \mathrm{~mm}$. The water content was varied from $0.5 \%$ to $12 \%$. The beads were wetted in a hermetic seal by mixing a mass of $0.1 \mathrm{~kg}$ of dry beads with the amount of water required to reach the targeted water content. The wetted particles were then moulded in a cylindrical vessel. Figure 8(a) shows a photo of a typical sample prepared according to this protocol.

Each cylindrical sample is subjected to axial compression up to failure. Figure 9 shows the compressive strength $\sigma_{\text {rupt }}$, corresponding to the axial stress at failure, as a function of water content.

\subsection{Numerical study}

4.2.1. Sample preparation The numerical samples are of cylindrical shape and composed of 8000 spherical particles with diameters from $0.8 \mathrm{~mm}$ to $1.3 \mathrm{~mm}$, as in experiments. The particle size grading is given in Table II.

\begin{tabular}{l|llllll}
\hline Diameter $(\mathrm{mm})$ & 0.8 & 0.9 & 1 & 1.1 & 1.2 & 1.3 \\
\hline Proportion $(\%)$ & 10 & 20 & 20 & 20 & 20 & 10 \\
\hline
\end{tabular}

Table II. Particle size grading in numerical samples

The samples are prepared by initially letting the particles fall into a cylindrical box of a diameter of $25 \mathrm{~mm}$. Then, the sample is "sealed" by adding an upper wall loaded vertically. At this point, the sample is $17 \mathrm{~mm}$ high and has a solid fraction of 0.62 . Finally, the capillary cohesion is introduced 


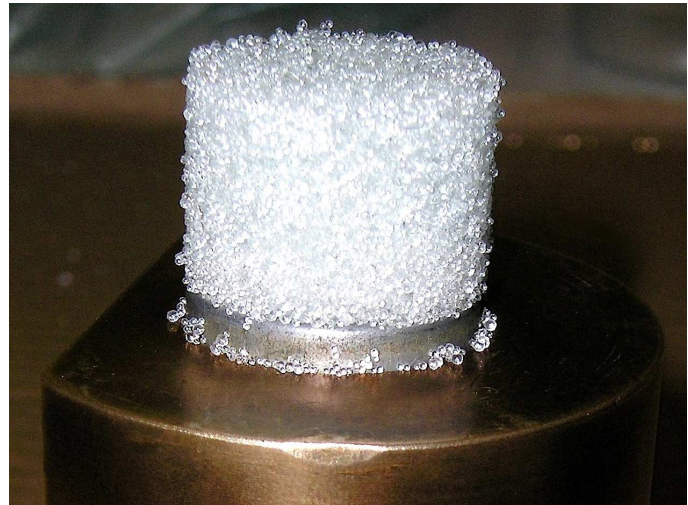

(a)

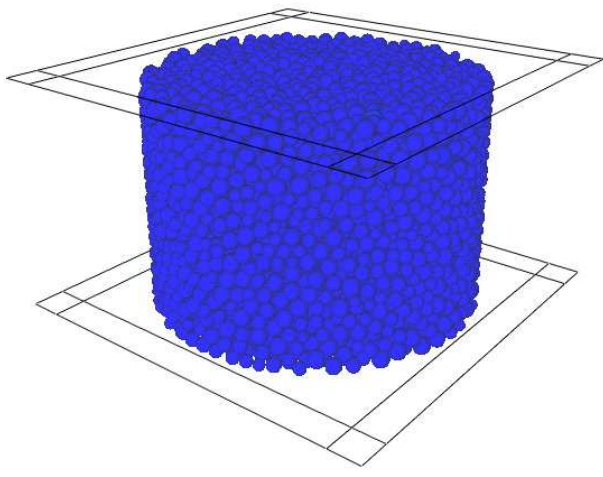

(b)

Figure 8. Typical sample used in simple compression tests. (a) Glass beads for $w=3 \%$, (b) Spherical particles in simulations.

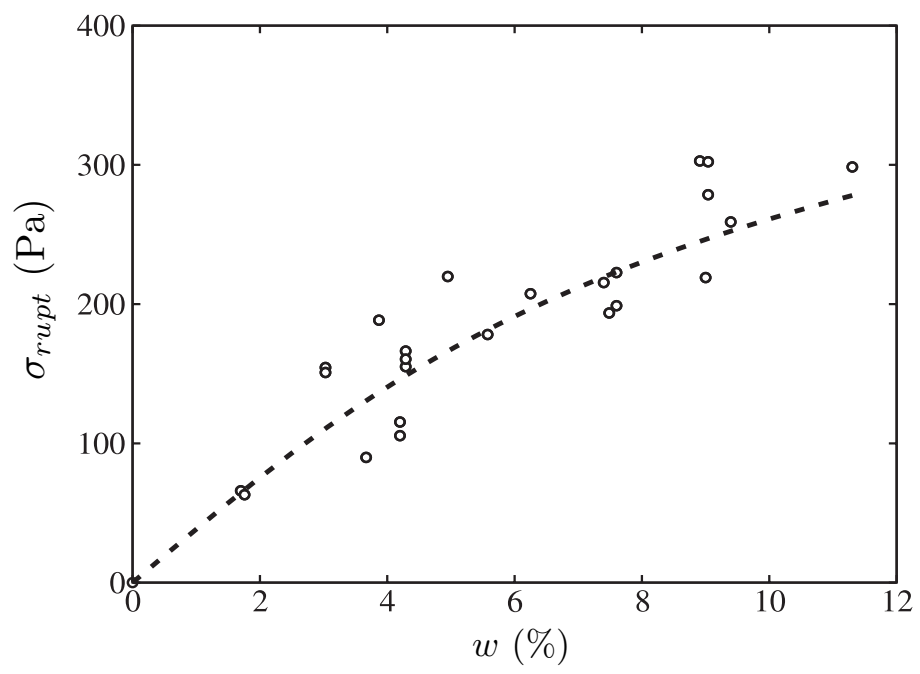

Figure 9. Compressive strength as a function of water content. Experimental data obtained by Soulié [41].

between eligible pairs of particles and the surrounding cylindrical wall is removed. The sample relaxes to equilibrium due to capillary cohesion with only a slight settlement of about $0.1 \mathrm{~mm}$ due to gravity; see Figure 8(b).

4.2.2. Numerical results and comparison with experiments Numerical simulations were carried out for $2 \%, 4 \%$ and $6 \%$ of water content. In each simulation, the upper wall moves at $1 \mathrm{~mm} / \mathrm{s}$ for 2 seconds. The axial stress $\sigma$ is simply estimated from the axial resultant of the forces acting on the upper wall divided by its area, as in experiments. The axial stress $\sigma$ is shown in Figure 10 as a function of axial shorting $\delta h$ normalized by the average particle diameter $\langle D\rangle$. The compressive strength $\sigma_{\text {rupt }}$ 
corresponds to the axial stress at the peak. Remark that the axial stress declines beyond the stress peak.

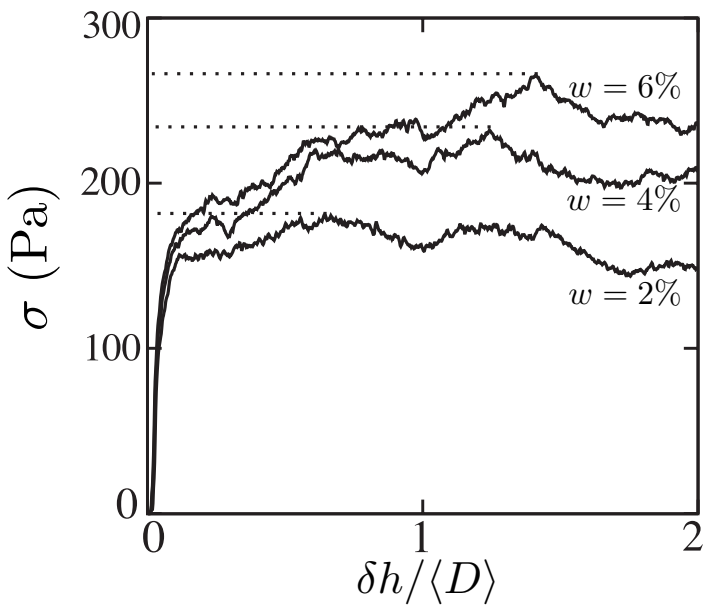

Figure 10. Axial stress as a function of axial strain for three different values of the water content.

In Figure 11 a snapshot of the sample of $w=2 \%$ is displayed at the end of the simulation. We observe the barrel shape of the sample, as observed in experiments with granular soils. Figure 11 shows a vertical section of the sample with color-coded particle displacements. We observe an immobile cone at the lower central part of the sample which can be attributed either to the motion of the upper wall or to the effect of gravity.

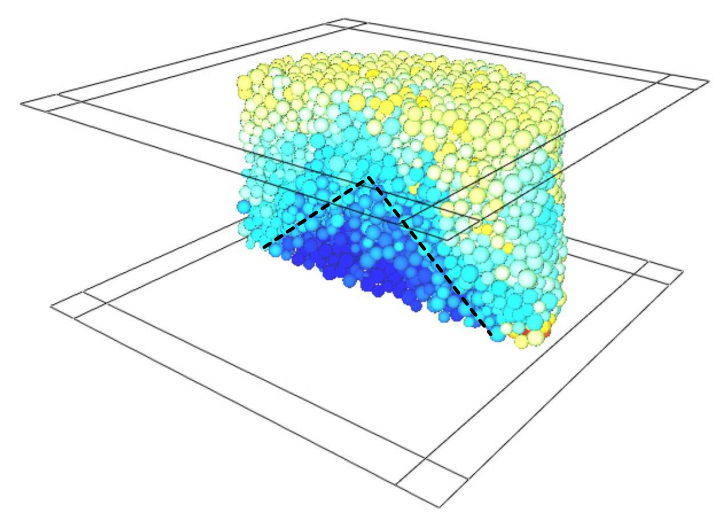

Figure 11. Numerical sample at the end of the simulation with $w=2 \%$. We observe a nearly immobile region indicated by dashed lines.

The compressive strengths are plotted as a function of water content in Figure 12 together with the numerical data of Soulié and the experimental data of Figure 9. We see that the numerical data of our simulations are closer to the experimental data than the initial numerical data of Soulié. It should be noted that the capillary law used by Soulié is different from the one employed in the present work. But 
the fits to the data obtained from direct integration of Young-Laplace equations are quite similar.

In order to find hints to explain the observed discrepancies, we carried out more simulations using different protocols for water redistribution. In addition to the two protocols introduced in Sections 2.4 and 3.2, we used another protocol in which the effect of gravity is accounted for by transferring the liquid from broken bond between two particles to the lowest contact located on the lower hemisphere of each of the two particles (Protocol 3). In this protocol, the volume of the liquid bond is shared by the two particles proportionally to their sizes. During shearing the liquid is gradually transported downward according to the bonding-debonding dynamics of the material. This dynamics being generally slow, the transport of liquid by this mechanism is not effective for small deformations.

Two points were checked in more detail: (1) the rule for the redistribution of water when a capillary bond fails according to the three protocols introduced in the preceding sections $(2.4,3.2,4.2)$, and (2) the bond coordination number $z$ which simply depends on the initial selection of eligible pairs of particles. In fact, as in the case of direct shearing, our data from simple compression show that the main influence comes from $z$. In order to illustrate this point, in Figure 12 two data pointsare shown from two simulations for $w=2 \%$ and $w=4 \%$ with reduced number of liquid bonds by a factor 2 . We see that the shear strength for this two samples is significantly reduced compared to the other simulations with different protocols.

On the other hand, we see that, as long as $z$ is the same, the protocols for water redistribution have nearly no effect. The difference between our simulations and those of Soulie is thus only related to the initial values of $z$. Indeed, in contrast to our simulations, Soulié did not allow the sample relax after removal of the cylinder used to mould the sample. The relaxation of the sample before compression leads to lower liquid bond coordination. In this respect, it should be noted that also in experiments water is not distributed to all present or eligible particle pairs. Shaking might be never efficient enough to allow all pairs to receive liquid bonds. Experimental investigation of water distribution seems thus to be crucial for a better modeling of wet granular media [21, 22].

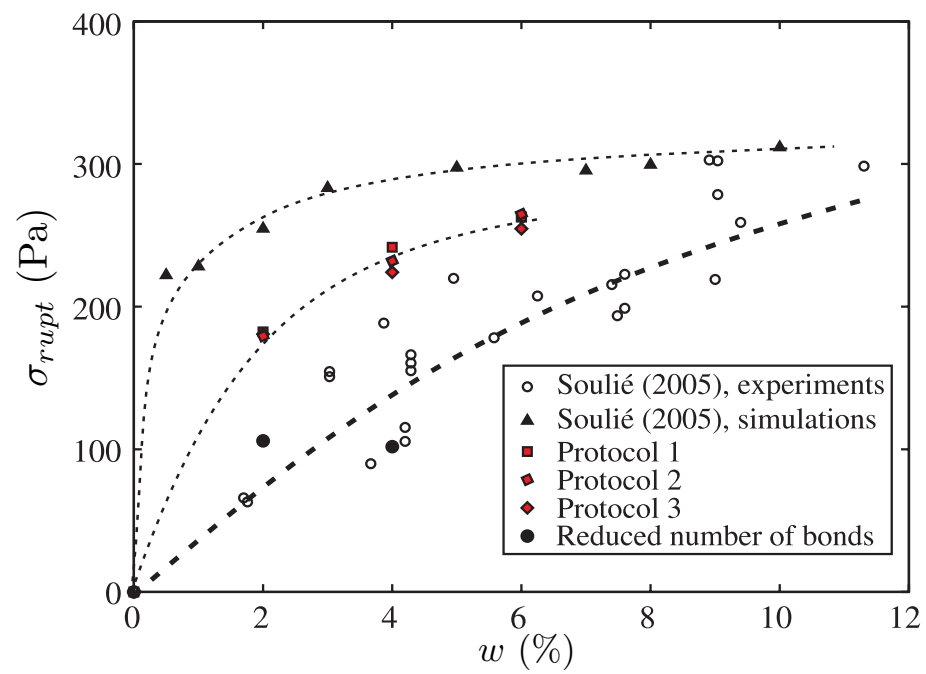

Figure 12. Compressive strengths as a function of liquid content in simple compression tests from experiments and simulations for three different redistribution protocols (see text). 


\section{CONCLUSION}

In summary, we developed a DEM-type approach for the simulation of 3D wet granular materials with spherical particles. The capillary law implemented in this framework is an analytical expression of the capillary force as a function of geometrical and materials parameters of a liquid bridge, and it was shown to provide excellent fit for the data from direct integration of Young-Laplace equations. This code (tapio-K, see [43]) was applied to simulate the quasi-static behavior of wet granular media for direct shear and simple compression boundary conditions and compared to simulations carried out with nearly the same parameters and boundary conditions. The experimental setup was specially designed to allow for the measurement of weak stresses, a necessary condition for the evaluation of the effects of capillary cohesion with millimeter-size particles.

Experimental direct shear tests were performed with glass beads and sand, and the shear strengths were analyzed in the Mohr-Coulomb space for weak confining stresses (below $1 \mathrm{kPa}$ ). We found that the internal angle of friction was not sensitive to water content $w$ and the Coulomb cohesion increased in a nonlinear fashion with $w$ to saturate to a well-defined level $c_{m}$ of cohesion independent of water content for $w>w_{m}$. A similar behavior was observed in numerical simulations. The cohesion $c_{m}$ was found to be quite close between the experiments and numerics for the samples of the same particle size distribution ( $c_{m}=120 \mathrm{~Pa}$ in numerics vs. $c_{m}=150 \mathrm{~Pa}$ in experiments). The numerical values of $w_{m}$ were found to be systematically below those in experiments.

For simple compression tests, we compared experimental results of Soulié [41] with numerical simulations performed with cylindrical samples of wet particles. Comparing the influence of water content between experiments and simulations, we found discrepancies that were analyzed by further simulations where the bond coordination number was varied. We found that, in contrast to water content, the bond coordination number plays a major role in compressive strength. Numerically, it was also shown that the details of the redistribution of water at failed capillary bonds has minor effect on the behavior.

Our findings and the comparisons made with experiments carried out with similar boundary conditions credits the numerical method, and more particularly the implemented capillary law. On the other hand, the observed discrepancies open new queries concerning the distribution of water in real samples of wet grains. It seems thus that, in order to progress in understanding the strength properties of wet granular materials, more detailed observations are required. New experiments are presently under way to evaluate the local distribution of capillary bridges and the influence of the mixing protocol.

\section{REFERENCES}

1. Cundall PA, Strack ODL. A discrete numerical model for granular assemblies. Géotechnique 1979; 29 : 47-65.

2. Thornton C. Computer Simulation of Impact Fracture/Fragmentation. In The first Nisshin Engineering Particle Technology International Seminar: Discrete Particle Simulations in Powder Technology. Osaka, Japan, 1993.

3. Moreau JJ. New computation methods in granular dynamics. In Powders \& Grains 1993; Thornton C (ed). AA Balkema, Rotterdam, 1993.

4. Jean M. The non-smooth contact dynamics method. In Computer Methods in Applied Mechanics and Engineering 1999; 117: $235-257$.

5. Luding S, Manetsberger K, Muellers J. A discrete model for long time sintering. Journal of the Mechanics and Physics of Solids. 2005; 53(2): 455-491.

6. Donzé F, Magnier SA. Formulation of a 3D numerical model of brittle behavior. Geophys. J. Int. 1995; 122: 790-802.

7. Radjaï F, Preechawuttipong I, Peyroux R. Cohesive granular texture. In Continuous and discontinuous modelling of cohesive frictional materials, Vermeer PA, Diebels S, Ehlers W, Herrmann HJ, Luding S, Ramm E (eds). Springer Verlag, 2000; 148-159. 
8. Delenne JY, El Youssoufi MS, Cherblanc F, Bénet JC. Mechanical behaviour and failure of cohesive granular materials. Int. J. Numer. Anal. Meth. Geomech. 2004; 28: 1577-1594.

9. Richefeu V, Radjaï F, El Youssoufi MS. Stress transmission in wet granular materials. European Physical Journal E 2006; 21: 359-369.

10. Bika DG, Gentzler M, Michaels JN. Mechanical properties of agglomerates. Powder Technology 2001; 117: 98-112.

11. Iveson SM, Beathe JA, Page NW. The dynamics strength of partially saturated powder compacts: the effect of liquid properties. Powder Technology 2002; 127: 149-161.

12. Thornton C, Ciomocos MT, Adams MJ. Numerical simulations of diametrical compression tests on agglomerates. Powder Technology 2004; 140: 258-267.

13. Lian G, Thornton C, Adams MJ. Discrete particle simulation of agglomerate coalescence. Chemical Engineering Science 1998; 53: 3381-3391.

14. Rhodes MJ, Wang XS, Nguyen M, Stewart P, Liffman K. Onset of cohesive behaviour in gas fluidized beds: a numerical study using DEM simulation. Chemical Engineering Science 2001; 56: 4433-4438.

15. Mikami T, Kamiya H, Horio M. Numerical simulation of cohesive powder behavior in fluidized bed. Chemical Engineering Science 1998; 53(10): 1927-1940.

16. Gröger T, Tüzün U, Heyes DM. Modelling and measuring of cohesion in wet granular materials. Powder Technology 2003; 133:203-215.

17. Jiang MJ, Leroueil S, Konrad JM. Insight into shear strength functions of unsaturated granulates by DEM analyses. Computers and Geotechnics 2004; 31(6): 473-489.

18. Pierrat P, Caram H. Tensile strength of wet granular materials. Powder Technology 1997; 91: 83-93.

19. Betz G, Bürgin PJ, Leuenberger H. Power Consumption Profile Analysis and Tensile Strength Measurements during Moist Agglomeration Process. International Journal of Pharmaceutics 2003; 252: 11-25.

20. Kim T, Hwang C. Modeling of tensile strength on moist granular earth material at low water content. Engineering Geology 2003; 69: 233-244.

21. Fournier Z, Geromichalos D, Herminghaus S, Kohonen MM, Mugele F, Scheel M, Schulz M, Schulz B, Schier C, Seemann R, Shudelny A. Mechanical properties of wet granular materials. Applied Physics: Condensed Matter 2005; 17: 477-502.

22. Kohonen MM, Geromichalos D, Scheel M, Schier C, Herminghaus S. On capillary bridges in wet granular materials. Physica A 2004; 339: 7-15.

23. Allen MP, Tildesley DJ. Computer Simulation of Liquids, Oxford Science Publications, 1987.

24. Maugis D. Contact, adhesion and rupture of elastic solids. Solid-State Sciences, Berlin, 1999.

25. Schäfer J, Dippel S, Wolf DE. Force schemes in simulations of granular materials J. Phys. I 1996; 6: 5-20.

26. Erle MA, Dyson DC, Morrow NR. Liquid bridges between cylinders, in a torus, and between spheres. AIChE J. 1971; 17: 115-121.

27. Lian G, Thornton C, Adams MJ. A theoretical study of the liquid bridge forces between two rigid spherical bodies. Journal of Colloid and Interface Science 1993; 161: 138-147.

28. Soulié F, Cherblanc F, El Youssoufi MS, Saix C. Influence of liquid bridges on the mechanical behaviour of polydisperse granular materials. Int. J. Numer. Anal. Meth. Geomech. 2006; 30: 213-228.

29. Willett CD, Adams MJ, Johnson SA, Seville JPK. Capillary bridges between two spherical bodies. Langmuir 2000; 16: 9396-9405.

30. Bocquet L, Charlaix E, Restagno F. Physics of humid granular media. C. R. Phys. 2002; 3: 207-215.

31. Herminghaus S. Dynamics of wet granular matter. Adv. Phys. 2005; 54: 221-261.

32. Israelachvili JN. Intermolecular and Surface Forces. Academic Press, London, 1993.

33. Richefeu V, El Youssoufi MS, Radjai F. Shear strength properties of wet granular materials. Physical Review E 2006; 73: 051304.

34. Dippel S, Batrouni GG, Wolf DE. Motion of a particle on a rough inclined plane: comparison of 2 and 3 dimensions. Physical Review E 1997; 56: 3645.

35. Luding S. In Physics of Dry Granular Media - NATO ASI Series E 350, Herrmann HJ, Hovi JP, Luding S (eds). Kluwer Academic Publishers: Dordrecht, 1998.

36. Halsey TC, Levine AJ. How sandcastels fall. Physical Review Letter 1998; 80(14): 3141-3144.

37. Hubbert MK. Mechanical basis for certain geological with recording of some of the experiments. This structures. Bulletin of the Geological Society of America 1951; 62: 355-372.

38. Schellart WP. Shear test results for cohesion and friction coefficients for different granular materials: scaling implication for their usage in analogue modelling. Tectonophysics 2000; 324: 1-16.

39. Rossi D, Storti F. New artificial granular materials for analogue laboratory experiments: aluminium and siliceous microspheres. J. Struct. Geol. 2003; 25: 1893-1899.

40. Van Mechelen J. Strength of moist sand controlled by surface tension for tectonic analogue modelling. Tectonophysics 2004; 384: 275-284.

41. Soulié F. Cohésion par capillarité et comportement mécanique de milieux granulaires. Ph.D. Thesis. Université Montpellier 2, France, 2005.

42. Rumpf H, Zur Theorie der Zugfestigkeit von Agglomerate bei Kraftübertragung an Kontaktpunkten. Chem.-Ing.-Tech. 1970; 42(8): 538-540. 
43. Richefeu V. Approche par éléments discrets 3D du comportement de matériaux granulaires cohésifs faiblement contraints $\mathrm{Ph}$.D. Thesis. Université Montpellier 2, France, 2005. 